Friedrich Müller* und Stefan Schmunk

\title{
Bedeutung und Potenzial von Geoinformationen und deren Anwendungen im Kontext von Bibliotheken und digitalen Sammlungen
}

https://doi.org/10.1515/bfp-2018-0049

Zusammenfassung: In den letzten Jahren ist ein gesteigerter Bedarf vieler Fachdisziplinen an bereitgestellten Geoinformationen und Forschungsanwendungen mit GISKomponente(n) zu verzeichnen. Im Hinblick auf die digitale Transformation von Bibliotheken und den damit verbundenen fortschreitenden technischen und informationswissenschaftlichen Möglichkeiten wie Methoden des Text und Data Mining stehen zudem viele raumzeitliche Informationen in Bibliotheken zur Verfügung. In diesem Kontext gibt dieser Artikel einen exemplarischen Überblick über bereits vorhandene Entwicklungen in Bibliotheken weltweit, nennt Perspektiven der Erschließung und Nutzung von Geoinformationen für einen gewinnbringenden, zukunftsorientierten Einsatz und zeigt auf, welche Komponenten und Services in Zukunft von Bibliotheken angeboten werden könnten.

Schlüsselwörter: Geoinformation; Digitale Transformation; GIS; Informationsmanagement

Importance and Potential of Geoinformation and its Applications in the Context of Libraries and Digital Collections

Abstract: In recent years, there has been an increased demand from many disciplines in providing geographic information and research applications with GIS component(s). With respect to the digital transformation of libraries and the associated technical advances and progress in information science, such as text and data mining methods, a large amount of spatiotemporal information is available in libraries. In this context, this article provides an exemplary overview of existing developments in libraries worldwide, identifies perspectives on the development and use of geoinformation for beneficial, future-oriented deployment, and names which components and services could be offered by libraries in the future.

*Kontaktperson: Friedrich Müller, friedrich.mueller@sub.uni-goettingen.de

Stefan Schmunk, stefan.schmunk@h-da.de
Keywords: Geoinformation; digital transformation; GIS; information management

Inhalt

1 Einleitung.................. 21

2 Derzeitiger Stand und Herausforderungen . . . . 23

2.1 Portale für raumzeitliche Daten in Bibliotheken . . 23

2.2 Forschungsanwendungen für raumzeitliche Daten

in Bibliotheken . . . . . . . . . . . . . 26

3 Perspektiven..................... 29

3.1 Datenmanagement ................ 29

3.2 Applikationen .................. 31

3.3 Support und Beratung . . . . . . . . . . . 32

4 Schlussbemerkung ............. 32

\section{Einleitung}

Die wichtigste internationale Fachmesse für das Tätigkeitsfeld Geoinformation in Deutschland, die INTERGEO, ${ }^{1}$ wird in diesem Jahr 2018 unter dem Slogan „Geoinformation - die DNA der Digitalisierung“ veranstaltet. Die Veranstalter vergleichen Geoinformationen mit Sequenzen des Erbguts und nutzen zugleich diese Metapher um zu verdeutlichen, dass Geoinformationen beim digitalen Wandel eine Steuerungsfunktion einnimmt, die nicht nur für Wirtschaft und Gesellschaft von hohem Nutzen ist, sondern auch für die Wissenschaft. ${ }^{2}$

Das Anwendungsspektrum von Geoinformationen ist vielschichtig und findet in den vergangenen Jahren in den unterschiedlichsten Fachdisziplinen vermehrt ihren Einsatz. Von stadtplanerischen Fragestellungen bis hin zu geografischen Analysen und Visualisierungen im Zusammenhang mit soziologischen, historischen und literari-

$1 \mathrm{http}: / /$ www.intergeo.de.

2 http://www.intergeo.de/media/docs/intergeo/2018/Presse/INTER GEO_Pressemeldung_Geoinformation_die_DNA_der_Digitalisierung. pdf. 
schen Studien. ${ }^{3}$ Jede Fachdisziplin, die Fragestellungen bzgl. der räumlichen Dimension umfasst bzw. thematisiert, nutzt Geografische Informationssysteme (GIS), um Informationen in verschiedenen Kontexten zu visualisieren als auch zu analysieren.

Bibliotheken sind Pioniere in der Sammlung und Bereitstellung von Informationen - Informationen zu erschließen, zu bewahren, diese vorzuhalten und ggf. Sammlungen zu generieren -, so dass Gesellschaft und Wissenschaft sie nutzen können, sind grundsätzliche Aufgaben.

Geoinformationen in Bibliotheksbeständen und -sammlungen - wie Kartensammlungen - sind zwar oftmals über Bibliothekskataloge oder Discovery-Systeme zugänglich, ein strukturierter und analytischer Zugriff per unterstützende Funktionen für Geodaten, wie mithilfe von interaktiven Kartenanwendungen, fehlt aber oftmals. Dies ist insofern verwunderlich, als dass die dafür notwendigen Informationen in einigen Fällen in standardisierter und strukturierter Form in der Regel bereits vorliegen und im Rahmen von Geoanwendungen effektiver genutzt und analysiert werden könnten. Solch eine Nutzung würde es erlauben, räumliche Zusammenhänge mit geeigneten Mitteln zu organisieren, zu präsentieren und damit die Forschung an den Daten gezielt zu unterstützen. ${ }^{4}$

In Bibliotheken dienen, neben zeitlichen und thematischen Angaben, räumliche Informationen oftmals zur Beschreibung und Wiederauffindbarkeit unterschiedlichster Medienformate. Bibliotheken besitzen dadurch einen reichhaltigen Schatz an Geoinformationen, sei es in Form von Sammlungen wie etwa von (historischen) Karten und Globen oder sei es durch geografisch verwertbare Metadaten von gedruckten Werken. Geografische Angaben sind nicht nur in den Provenienzinformationen enthalten, sondern sind auch zahlreich in den Texten selbst zu finden. Ein geografischer vielfältiger Schatz, der bislang nur selten gehoben und den entsprechenden wissenschaftlichen wie auch öffentlichen Communities zur Verfügung gestellt wird. Zudem bieten erschlossene Volltexte mit Ortsentitäten eine Vielzahl an neuen Suchmöglichkeiten und stellen den Ausgang für eine auf Geoinformationen beruhende semantische Vernetzung der Korpora dar. ${ }^{5}$ Verbunden mit Zeitinformationen können hierdurch neue Formen von raumzeitlichen Analysen betrieben und durch elektronische Werkzeuge und neue methodische Ansätze nun

3 Godfrey (2016) 29.

4 Michalec und Welsh (2007) $66 \mathrm{ff}$.

5 Vgl. Leidner et al. (2003). erstmals Zeit-Raum-Bezüge von Korpora und Sammlungen hergestellt werden.

Im digitalen Zeitalter sind Bibliotheken einem Paradigmenwechsel unterworfen, der dazu führt, dass nicht mehr die Dokumente nur als Informationsträger im Fokus stehen, sondern deren Inhalt und deren kontextuelle Verknüpfung der Dokumente selbst wissenschaftlicher Gegenstand werden können. Kontextinformation geben Auskünfte über die Provenienz eines Dokumentes und externe Informationen erlauben die Interpretation. ${ }^{6}$ Dadurch verschwimmen die Grenzen zwischen Metadaten und Daten und die Rolle der Bibliotheken wechselt von einer reinen „Anbieteraufgabe“ von Dokumenten und Metadaten hin zu einem „Informations-Broker“. 7

Angesichts dieses Paradigmenwechsels und der fortschreitenden technischen und informationswissenschaftlichen Möglichkeiten wird eine Aufgabe von Bibliotheken in Zukunft darin bestehen - so unsere These -, dieses Potenzial für ihr Portfolio verstärkt zu erschließen und den wissenschaftlichen Communities neben dem technisch sowie lizenzrechtlich reibungslosen Zugang zu ihren Daten und Sammlungen mittels offener und standardisierter Schnittstellen zu generieren und ggf. auch entsprechende Forschungswerkzeuge als digitale Services anzubieten, so dass die bereitgestellten Daten medienbruchfrei nutzbar sind.

Geoinformationssysteme sind in dieser Perspektive eine Möglichkeit, um Fachwissenschaftler sowie Forschungseinrichtungen nicht nur adäquate technologische Zugänge zu den raumzeitlichen Informationen zu ermöglichen, ${ }^{8}$ sondern - von dieser Grundprämisse gehen wir aus - diese gemeinsam zwischen Bibliotheken und Wissenschaftlern, also nutzerbasiert und forschungsfokussiert, $\mathrm{zu}$ entwickeln und zu betreiben. Vielleicht mag dies als Vision interpretiert werden, aber einige bereits existierende Dienste, wie beispielsweise EarthWorks der Stanford Libraries, ${ }^{9}$ mit dem interaktive Zugangsarten zu wissenschaftlichen Datensätzen mit Raumbezügen ermöglicht werden, zeigen, dass es machbar ist. Ein weiteres Beispiel, wie geografische Informationen in anderer Ausprägung aufbereitet werden können, ist die Kartensammlung der British Library, in der mittlerweile mehr als 4,5 Millionen Karten, Pläne und Stiche elektronisch zugänglich sind. ${ }^{10}$ Die British Library setzt dabei nicht auf eine eigene WebGIS-Lösung, sondern veröffentlicht ihren georeferenzier-

6 Vgl. Gradmann (2014) 241.

7 Scheider et al. (2014) 139.

8 Vgl. Michalec und Welsh (2007).

9 https://earthworks.stanford.edu.

10 https://www.bl.uk/subjects/maps. 
ten Kartenbestand ${ }^{11}$ über das Kartenportal Old Maps Online. ${ }^{12}$

Im Rahmen dieses Beitrages stehen deshalb zwei zentrale Fragen:

1. Welche Anwendungen für Geoinformationen werden von Bibliotheken angeboten und welche raumbezogenen Zugänge zu bibliothekarischen Daten und Sammlungen existieren bereits?

2. Welche Perspektiven der Erschließung und Nutzung sind für einen gewinnbringenden, zukunftsorientierten Einsatz denkbar und welche Komponenten könnten von Bibliotheken im Rahmen ihrer Geodienste angeboten werden?

Zuvor eine Prämisse vorab. Für die skizzierten neuen Herausforderungen hinsichtlich der wachsenden Verfügbarkeit von raumzeitlichen Daten müssen diese entsprechend nachhaltig aufbereitet, über geeignete Portale recherchierbar und über offene, webbasierte Schnittstellen der Allgemeinheit wie auch den wissenschaftlichen Communities zur Verfügung gestellt werden. Um zu verdeutlichen, wie die Entwicklungen der vergangenen Jahre in diesem Themenbereich verlief und welche weiteren Entwicklungen sich möglicherweise daraus abzeichnen lassen, wurde bewusst innerhalb dieses Artikels der Zugang gewählt, zuerst den Status quo exemplarisch zu beschreiben. Anschließend werden hinsichtlich weiterführender Entwicklungen im Abschnitt Perspektive die Umsetzungsmöglichkeiten in den Bereichen Datenmanagement, Applikationen als auch Support und Beratung beschrieben und Beispiele für potentielle Herangehensweisen aufgeführt.

Es wird kein Anspruch auf eine vollständige Evaluation und Darstellung erhoben, sondern dieser Artikel stellt vielmehr den Versuch dar, die wissenschaftlichen und fachöffentlichen Diskussionen der letzten Jahre mit den tatsächlich entwickelten und betriebenen Angeboten abzugleichen. Die im Folgenden genannten Beispiele sollen deshalb auch vielmehr verdeutlichen, mit welchen Themen, Angeboten und auch wissenschaftlichen Konzepten und Analysen wir uns im Rahmen der weiteren Entwicklung von GIS-Systemen an der SUB Göttingen beschäftigt haben, um Potenziale als auch Chancen für den Aufbau daten- und nutzerzentrierter Dienste zu eruieren.

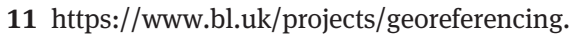
12 http://www.oldmapsonline.org.

\section{Derzeitiger Stand und Herausforderungen}

\subsection{Portale für raumzeitliche Daten in Bibliotheken}

Im heutigen Informationszeitalter sind Bibliotheken, mit ihren Kernfähigkeiten der Sammlung, Generierung, Verwaltung und Verbreitung von Wissen, zentrale Akteure der Digitalisierung. ${ }^{13}$ Durch den gesteigerten Bedarf an fachspezifischer Expertise und Anwendungen für Geoinformationen wie auch der hierfür notwendigen technischen (Forschungs-)Infrastrukturen wird seit dem vermehrten Einzug von GIS-Anwendungen in Bibliotheken in den frühen 1990er-Jahren das Service-Angebot größtenteils kontinuierlich weiterentwickelt, um die unterschiedlichen Nutzungsbedürfnisse zu decken. ${ }^{14}$

Der Aufgabenbereich der Bibliotheken ist in diesem Zuge ebenfalls mit der Bereitstellung von GIS-Diensten gewachsen. Hierzu zählen die Bereitstellung von raumzeitlichen Datensammlungen, die Realisierung von technischen Lösungen zur Verwaltung von geografischen Daten und Daten-Explorations-Tools und den dazugehörigen Support und Maßnahmen für einen Wissenstransfer. Diese Entwicklung zeigte sich in Projekten wie der Alexandria Digital Library (ADL), die das Ziel verfolgte, mit einer umfassenden Palette von Dienstleistungen für Sammlungen von Karten, Bildern und weiteren raumbezogenen Informationen, ein benutzerfreundliches digitales Bibliothekssystem zu entwickeln..$^{15}$ Auf deutscher Seite können als Beispiele sammlungsübergreifende Projekte wie GEO$\mathrm{LEO}^{16}$ und IKAR ${ }^{17}$ bzw. Fachinformationsdienste wie der Fachinformationsdienst Geowissenschaften der festen Erde (FID GEO), ${ }^{18}$ eine Kooperation des GeoForschungsZentrums Potsdam (GFZ) und der Niedersächsischen Staatsund Universitätsbibliothek Göttingen (SUB), und der Fachinformationsdienst Kartografie und Geobasisdaten (FIDKarten) ${ }^{19}$ der Kartenabteilung der Staatsbibliothek zu Berlin - Preußischer Kulturbesitz aufgeführt werden.

Auf Anwendungsebene sind ebenfalls in Deutschland nennenswerte Entwicklungen entstanden. Das Virtuelle

13 https://www.bmbf.de/de/bibliotheken-sind-zentrale-akteureder-digitalisierung-4520.html.

14 Weimer et al. (2012) $39 \mathrm{f}$.

15 Vgl. Goodchild (2004).

16 https://geo-leo.de.

17 http://ikar.staatsbibliothek-berlin.de.

$18 \mathrm{http}$ ///www.fidgeo.de.

$19 \mathrm{http} / /$ /kartografie.staatsbibliothek-berlin.de. 
Kartenforum 2.020 der Sächsischen Landesbibliothek Staats- und Universitätsbibliothek Dresden (SLUB), das über die reine Präsentation von Kartendigitalisate innerhalb einer Portalanwendung hinausgeht und Ansätze für eine Geodateninfrastruktur (GDI) mit standardisierten Schnittstellen ${ }^{21}$ und interaktivem Web-GIS bietet.

Neben reinen Kartenportalen, wie dem Kartenportal. $\mathrm{CH},{ }^{22}$ ein Projekt mit Beteiligung der Zentralbibliothek Züri ch und Lib4RI: Library of Eawag, Empa, WSL \& PSI, Düben dorf, entstanden Geoinformations-Services, die es erlauben, Kartendigitalisate, Bilder und Dokumente vernetzt durch den geografischen Bezug recherchierbar $\mathrm{zu}$ machen.

Die innovative Anwendung ETHorama ${ }^{23}$ der ETH-Bibliothek ${ }^{24}$ in Zürich ermöglicht durch eine interaktive Karte ein Recherche-Tool zu den Digitalisaten, die mit einem bestimmten Ort geografisch miteinander verbunden sind. Zudem erlaubt die Funktion „Historische Reisen“,25 die Darstellung von Reiseberichten berühmter Persönlichkeiten, die Orte damit in der Schweiz historisch erfahrbar machen. Die Anwendung ETHorama ist gekoppelt mit dem Wissensportal ${ }^{26}$ der ETH-Bibliothek und stellt Empfehlungen für verknüpfte Dokumente und relevante Orte bereit, die zu einer Verbesserung der Suchergebnisse führen.

Weiterhin gibt es Portale, die nicht nur Dokumente, Bilder und Karten beinhalten, sondern auch GIS-spezifische Datentypen wie Punkte, Linien und Polygone in unterschiedlichen fachbezogenen Sammlungen, beispielsweise der Geologie oder der Demografie verwalten. Derartige Portale erlauben Bibliotheken ihre Rolle als Informations-Broker auch in Bezug auf Geodaten wahrzunehmen. So gibt es in unseren unmittelbaren Nachbarländern wie der Schweiz Initiativen - der Service geodata4edu.ch ${ }^{27}$ mit Beteiligung der ETH-Bibliothek ${ }^{28}$ ist hier exemplarisch zu nennen -, die Geodaten für Lehre und Forschung zur Verfügung stellen.

Auch in den USA findet man an Bibliotheken spezifische Portale für Geodaten. Als Beispiele können die Entwicklungen MIT GeoWeb ${ }^{29}$ der Massachusetts Institute of

20 http://kartenforum.slub-dresden.de/.

21 Beispielsweise Open Geospatial Consortium (OGC)-konforme Service-Schnittstellen wie Web Map Service (WMS).

$22 \mathrm{http://www.kartenportal.ch.}$

$23 \mathrm{http} / / /$ ethorama.library.ethz.ch/de.

24 Eidgenössische Technische Hochschule Zürich.

25 http://ethorama.library.ethz.ch/de/routes.

$26 \mathrm{http}: / /$ www.library.ethz.ch/de/.

27 https://www.geodata4edu.ch.

28 http://www.library.ethz.ch/Ressourcen/Geodaten-Karten.

29 https://arrowsmith.mit.edu/mitogp.
Technology (MIT) Libraries, Spatial Data Explorer ${ }^{30}$ der Arizona University Libraries, EarthWorks ${ }^{31}$ von Stanford Libraries $^{32}$ und die Geodaten-Sammlung der NC State University Library ${ }^{33}$ genannt werden.

Weiterhin sind bei Bibliotheken im Bereich der Geodaten auch ihre originären Aufgaben der Sammlung und Erhaltung gefragt. Das National Geospatial Digital Archive (NGDA) ${ }^{34}$ beschäftigte sich zwischen 2005 bis 2009 mit der Fragestellung, wie Geodaten im Rahmen eines landesweiten, US-amerikanischen, föderierten Archivs, das von mehreren Partnern und Einrichtungen getragen werden sollte, für die Nachwelt erhalten werden können. ${ }^{35}$ Hierbei wurden zwar eine Reihe von Empfehlungen und Policies entwickelt, ${ }^{36}$ das Projekt ist aber in den letzten Jahren nach Ende der Förderung im Jahre 2009 nicht mehr öffentlich aktiv geworden und die Frage, ob und in welchem Umfang dessen Empfehlungen angewandt wurden bzw. heute noch werden, deshalb nicht evident prüfbar.

Auf deutscher Seite kann die Anwendung GFZ Data Services ${ }^{37}$ der Sektion Bibliothek und Informationsdienste (LIS) des GFZ Potsdams als ein Service-Beispiel für die Verwaltung von Geoinformationen genannt werden. GFZ Data Service erlaubt das Publizieren und Beziehen von Geodaten aus allen geowissenschaftlichen Disziplinen per Digital Object Identifiers (DOI) über ein Portal, das die Geodaten mittels interaktiver Kartenanwendung visualisiert und zur Suche bereitstellt. Diese Geodaten können dann mit Publikationsdaten gekoppelt werden. Ein Beispiel ist hier der FID GEO, der elektronisches Publizieren mit eindeutig referenzierten Geodaten erlaubt. Gerade die Funktionalität der persistenten Referenzierbarkeit ist in diesem Kontext hervorzuheben, da dadurch sichergestellt wird, dass die Geodaten sowohl machinenlesbar als auch klassisch zitierbar vorgehalten werden und so von Forschung und Wissenschaft genutzt werden können. Ein weiterer Vorteil ist, dass auf diese Weise Daten unmittelbar und persistent in Publikationen zitiert werden können, so dass die wissenschaftlichen Nutzer nicht nur die Forschungsergebnisse rezipieren können, sondern zugleich auch die Datenbasis selbst lesen und für eigene Forschungen nutzen können.

\footnotetext{
$30 \mathrm{https}$ //geo.library.arizona.edu. $31 \mathrm{https://earthworks.stanford.edu.}$ $32 \mathrm{http}: / /$ library.stanford.edu. $33 \mathrm{http://www.lib.ncsu.edu/gis.}$ 34 http://www.ngda.org/home.html, eine Initiative zur National Digital Information Infrastructure and Preservation.

35 Vgl. Janée (2009).

36 http://www.ngda.org/policies.html.

37 http://dataservices.gfz-potsdam.de/portal.
} 


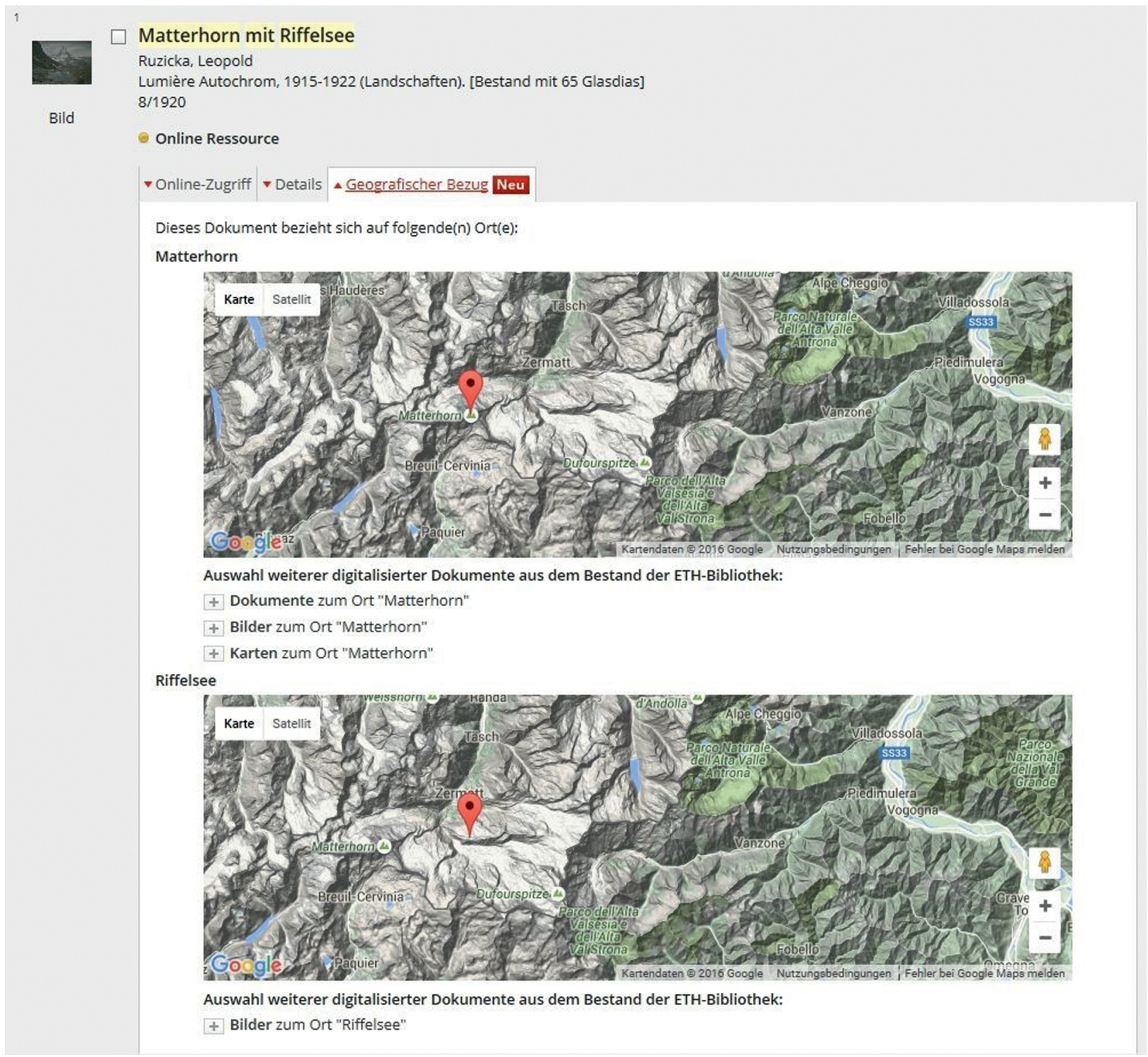

Abb. 1: Suche „Matterhorn mit Riffelsee“ im Wissensportal der ETH-Bibliothek, Kartenvisualisierung der Orte mit Empfehlungen für relevante Dokumente, Bilder und Karten. ${ }^{38}$

Als weiteres Beispiel ist der FID Karten ${ }^{39}$ zu nennen, dessen Services projektbezogene Kartenerwerbungen, thematische fokussierte Digitalisierungen, Geodatenvermittlung und das Angebot von Fachrepositorien und elektronischer Fachliteratur umfassen..$^{40}$ Anhand der ServiceBeispiele FID GEO und FID Karten wird das wachsende

38 http://blogt.ethz.ch/innovethbib/2016/07/12/wissensportal-ethor ama-ressourcen-georeferenziert-und-gruppiert.

$39 \mathrm{http} / / /$ kartografie.staatsbibliothek-berlin.de.

$40 \mathrm{http} / / /$ staatsbibliothek-berlin.de/sammlungen/sondersammelgebiete-und-fachinformationsdienste/kartografie-und-geobasisdaten/information.
Aufgabenspektrum und die Möglichkeiten des Einsatzes von Geoinformationen durch Bibliotheken im e-ScienceBereich sehr deutlich. Diese Bibliotheks-Services unterstützen Wissenschaftler nicht nur mit dem Bereitstellen von geo-relevanten Ressourcen, sondern auch beim Erwerb und der Digitalisierung von bisher nicht erschlossenen Materialien. Zudem werden Bibliotheken ihrer Rolle nicht nur als reiner Daten- und Informationsprovider gerecht, sondern auch als zentraler Wissensbroker, indem gezielt Beratungsangebote für die verschiedenen Fachbereiche initiiert werden. Hierfür ist die Beschaffung von Geobasisdaten und das Angebot von fachkompetenter Beratung, etwa hinsichtlich der Auswahl von geeigneten Da- 
ten und Formaten und die Vermittlung von weiterführenden Kontakten zu externen Geodatenanbietern, durch den FID Karten ein innovatives Beispiel, was hoffen lässt, dass vergleichbare Angebote zukünftig vermehrt initiiert und angeboten werden.

Der Einsatz von spezifischen Geodatenportalen erlaubt es zudem mehrere kritische Punkte der Suche und Distribution von Geoinformationen zu lösen, so u.a.:

1) Nach Geografika kann intuitiver und effektiver, beispielsweise durch Einsatz einer interaktiven Kartenanwendung, gesucht werden. Vernetzte räumliche Informationen erlauben nicht nur die ortsbezogene Ressourcen-Suche, sondern machen die Exploration von räumlichen Beziehungen möglich. ${ }^{41}$

2) Es wird eine einheitliche Lösung, für die unterschiedlichen Geografika wie Kartendigitalisate, sonstige Raster- und Vektordaten sowie Texte und Werke erstellt, womit Bibliotheken ihre geografischen Ressourcen zentral verwalten können. Um Insellösungen zu vermeiden, können die Ergebnisse mit bestehenden Services von Digitalisierungszentren bzw. Online-Katalogen verbunden werden. Zudem erhöht die Einbindung in übergeordnete Sammlungen, wie Europeana Libraries $^{42}$ für Europa bzw. andere weltweite Ansätze wie World Digital Library ${ }^{43}$, die Wiederverwendung. Sinnvoll erscheint auch die Vernetzung mit lokalen Geoportalen zu sein, wie es sich am Beispiel Virtuelles Kartenforum 2.0 im Zusammenspiel mit dem Geoportal Sachsenatlas ${ }^{44}$ zeigt.

3) Nur wenige Forschende werden im Gegensatz zu Bibliothekaren und Informationswissenschaftlern etwas mit einem MARC-Datensatz oder anderen bibliothekarischen Formaten anfangen können. ${ }^{45}$ Geodaten können in gängigen GIS-Datenformaten zum Beispiel als Shape-Datei oder GeoTIFF zur Verfügung gestellt werden. Geo-spezifische Metadaten-Standards wie ISO 19115 und ISO 19139 garantieren die Interoperabilität mit weiteren Geoanwendungen. ${ }^{46}$

4) Die Verfügbarkeit und Einbindung von Geodaten in externe Anwendungen kann über webbasierte, offene und standardisierte Schnittstellen, beispielsweise per

41 Z.B. „Zeige mir alle benachbarten Objekte im Umkreis von $100 \mathrm{~km}$ an“ oder "Zeige mir alle Objekte A an, die in B enthalten sind“.

42 https://pro.europeana.eu/project/europeana-libraries.

$43 \mathrm{https} / / /$ www.wdl.org/en.

44 https://geoportal.sachsen.de/cps/metadaten_portal.html?id=a15 0c87f-531a-4393-9454-8ed009b352b4.

45 Horstmann et al. (2015) 75.

46 Muratori und Samakovlija (2015) 169.
Open Geospatial Consortium (OGC) ${ }^{47}$-Standards, verbessert werden.

Hilfreich bei der Entwicklung von institutionellen Anwendungen für Geodaten sind ebenfalls Projekte, die kollaborativ Geodaten-Spezialisten, Softwareentwickler, Metadaten-Experten und Bibliothekare zusammenbringen. Hier kann als Beispiel das Projekt Open Geoportal (OGP) ${ }^{48}$ genannt werden, das eine Kollaboration aus verschiedenen Partnern u.a. MIT, Harvard und Tufts University ist. OGP entwickelt Open-Source-Portal-Lösungen und teilt diese in Verbindung mit dokumentierten Best Practices mit der Community.

\subsection{Forschungsanwendungen für raumzeitliche Daten in Bibliotheken}

Die Bedürfnisse von Forschung und Wissenschaft sind Trigger für forschungsrelevante Dienste auf Seiten der Bibliothek. ${ }^{49}$ Bibliotheken bieten seit jeher Informationsquellen, die zur Beantwortung von raumzeitlichen Forschungsfragen dienen. Aktuelle sowie historische raumzeitliche Informationen, in Form von digitalen Karten etwa, sind für eine Vielzahl von Forschungsfeldern von Bedeutung, so u. a. in der Epidemiologie, der Stadt- und Landschaftsentwicklung und der Geologie. ${ }^{50}$

Die damit verbundenen Publikationen haben spezifische Forschungsdaten und besitzen individuelle Anforderungen an deren Datenmanagement. Hierbei spielen raumzeitliche Informationen eine zentrale Rolle. ${ }^{51}$

Bei den Geistes- und Kulturwissenschaften sind generell textbasierende raumzeitliche Informationen im Fokus, vermehrt kommen durch die Digital Humanities interdisziplinäre Ansätze mit Methoden der Geoinformatik zum Tragen. Ein Beispiel ist der Ansatz der Kritischen Geodatenanalyse von Elrick und Kremer (2013) ${ }^{52}$ bei der die Wechselbeziehungen zwischen informationstechnischer und geistes- und sozialwissenschaftlicher Herangehensweise diskutiert werden. Hier verbinden sich Ansätze der Digital Humanities mit Geografie und Geoinformatik. Ein weiteres Projektbeispiel ist das Verbundvorhaben „RiR - Relationen

$47 \mathrm{http}: / /$ www.opengeospatial.org.

$48 \mathrm{http}$ ://opengeoportal.org.

49 Ceynowa (2018) 3.

50 Vgl. Pinol (2018).

51 Scheider et al. (2014) 139.

52 http://dhd-wp.hab.de/?q=content/kritische-geodatenanalyse-\% E2\%80\%93-ein-tragf\%C3\%A4higer-ansatz-f\% $\%$ C3\%BCr-die-digital-hu mantities-zwischen. 
im Raum “ ${ }^{53}$ Hier wurde eine Sammlung mit epigrafischen Informationen zu Grabmalen auf jüdischen Friedhöfen aus neun Jahrhunderten (11.-20. Jh.) als Grundlage für einen „Topografie-Visualizer“ verwendet. Dieser interdisziplinäre Ansatz verfolgte das Ziel, sozial- und kulturgeschichtliche Fragestellungen $\mathrm{zu}$ beantworten, um basierend auf topografischen Klein(st)strukturen zu analysieren, ob Geoinformationen der Grablagen Rückschlüsse auf gesellschaftliche Strukturen und Veränderungen über einen Zeitraum von neun Jahrhunderten geben können. ${ }^{54}$

Im Zuge des Engagements von Bibliotheken im Bereich Forschungsinfrastrukturen sind in zahlreichen Projekten Anwendungen für Geoinformationen entstanden. Im Bereich der Digital Humanities ermöglicht der DARIAHDE-Geo-Browser ${ }^{55}$ die Exploration von raumzeitlichen Daten. CLARIN-D besitzt im Rahmen von WebLicht ${ }^{56}$ ebenfalls eine Geovisualisierungs-Komponente, die eine kartenbasierte Darstellung von Entitäten eines Input-Textes erlaubt.

Das Projekt Geo Data Node (GDN), ${ }^{57}$ mit Beteiligung der Bibliothekssektion des GFZ Potsdam, beschäftigt sich mit der Thematik des nachhaltigen Datenmanagements und erarbeitet unterstützende Maßnahmen zur Umsetzung der FAIR-Prinzipien ${ }^{58}$ mit Fokus auf räumliche Daten, wobei die Erfahrungen der GFZ Data Services mit einfließen.

Weitere Projekte mit naturwissenschaftlichen Bezug wie der German Federation for the Curation of Biological Data (GfBio) ${ }^{59}$ erlauben mit Web-GIS-Lösungen wie „The Visualization, Analysis and Transformation System“ $(\mathrm{VAT})^{60}$ das Visualisieren und Analysieren von heterogenen Forschungsdaten aus verschiedenen Fachbereichen wie Geografie, Umweltwissenschaften sowie Sammlungsdaten im raumzeitlichen Kontext. Bei der Entwicklung der serviceorientierten Dateninfrastruktur sind Bibliotheken wie die SUB Göttingen und die Universitätsbibliothek der Freien Universität Berlin beteiligt. ${ }^{61}$

53 http://www.steinheim-institut.de/wiki/index.php/RiR\#Relatione n_im_Raum._Visualisierung_topografischer_Klein.28st.29strukturen. $54 \mathrm{https}$ ://spacehumanities.de.

55 https://geobrowser.de.dariah.eu.

56 https://www.clarin-d.de/de/sprachressourcen-und-dienste/we blicht, https://weblicht.sfs.uni-tuebingen.de/weblichtwiki/index.ph p/Tools_in_Detail\#Geovisualization.

57 https://www.gfz-potsdam.de/wissenschaftliche-infrastruktur/bi bliothek-und-informationsdienste/projekte/gdn-geo-data-node.

58 Findable, Accessible, Interoperable, Reusable (FAIR).

59 https://www.gfbio.org.

$60 \mathrm{https} / / /$ www.gfbio.org/data/visualizeandanalyze.

$61 \mathrm{https} / / /$ www.sub.uni-goettingen.de/projekte-forschung/projektdetails/projekt/gfbio und https://www.sub.uni-goettingen.de/projek te-forschung/projektdetails/projekt/gfbio-ii.
Angesichts der fortschreitenden Entwicklung der automatischen Auswertung und Analyse von Texten und der damit massenhaften Verfügbarkeit von Orts- und Zeit-Entitäten durch Bereiche der Data Science wie Text Mining, Named Entity Recognition (NER) und Machine Learning sind innovative Herangehensweisen notwendig, die diese raumzeitlichen Analysen und die Geovisualisierung der Orte per GIS ermöglichen. ${ }^{62}$ Der Edinburgh Geoparser ${ }^{63}$ ist solch eine Entwicklung mit NER-Komponente, mit der man sich automatisiert Ortsentitäten in Texten ermitteln, disambiguieren und per Karte visualisieren kann. Eine Herangehensweise per Machine Learning, unter Verwendung der entsprechenden Modelle, ermöglicht ebenfalls eine effiziente Erkennung von Ortsreferenzen in Texten und deren Disambiguierung. ${ }^{64}$

Gerade im Bereich der GeoHumanities bzw. Spatial Humanities, ein interdisziplinärer Ansatz, der zwischen Konzepten und Methoden der Geografie, Geoinformatik und den Geistes- und Kulturwissenschaften ${ }^{65}$ angesiedelt ist, sind textbasierte Anwendungen von großem Interesse. Ein Beispiel hierfür ist das CosmoTool, ${ }^{66}$ ein generisches Tool, das biografische Informationen aus strukturierten Daten (Metadaten) und unstrukturierten Daten (Fließtext) in „Quadruples“ (personale, temporale Entitäten mit einem Ortsbezug) aggregiert und raumzeitliche Informationen beispielsweise bzgl. der Mobilität per Kartenvisualisierung analysierbar macht. Zu diesem Komplex zählt auch der literarische Atlas Europas ${ }^{67}$ der ETH Zürich, der literarische Räume per Kartenvisualisierung zugänglich und untereinander vergleichbar macht.

In Verbindung mit der zahlreichen Bereitstellung von raumzeitlichen Informationen aus textbasierten Quellen ergeben sich neue Aufgabenfelder wie die Erarbeitung von anwendungsspezifischen Lösungen für Geoparsing im Kontext von historischen Gazetteers ${ }^{68}$ und den damit auftretenden Problemstellungen wie der Disambiguierung von Ortsnamen. ${ }^{69}$ Aber nicht nur das Identifizieren der räumlichen Information und das automatische Versehen mit Geokoordinaten stehen im Fokus, sondern auch das Informationsmanagement mit semantischen Technologien wie Linked Data. ${ }^{70}$ Zahlreiche Bibliotheken und Biblio-

62 Vgl. Won et al. (2018).

63 http://jekyll.inf.ed.ac.uk/geoparser.html.

64 Vgl. Martins et al. (2010).

65 Vgl. Dear (2011).

66 https://cosmotool.de.dariah.eu/cosmotool.

67 http://www.literaturatlas.eu/en.

68 Vgl. Goodchild und Hill (2008) und Alex et al. (2015).

69 Vgl. Overell und Rüger (2008).

70 Simon et al. (2015) 53. 
theksverbünde veröffentlichen ihre digitalen Bestände bereits als Linked Open Data, so die schwedische, ${ }^{71}$ deutsche, ${ }^{72}$ französische ${ }^{73}$ und ungarische ${ }^{74}$ Nationalbibliothek, die British Library ${ }^{75}$ und die Library of Congress. ${ }^{76}$ Linked Data verbessert generell das Management und die Auffindbarkeit von Medien auf Basis der Verknüpfung von Raum, Zeit und Semantik mit Inhalten. Besonders im Bereich der beschreibenden Metadaten bietet raumzeitliche Information verknüpft durch Linked-Data-Technologien die Möglichkeit, bestehende Bibliotheks-Services zu erweitern und neue Services zu erschließen. Die Integration und Anreicherung von semantischen Inhalten in Verbindung mit der Unterstützung von raumzeitlichen Referenzsystemen und unterschiedlichen Schemata in Bibliothekskatalogen verbessert die derzeitige Datennutzung, indem es den Zugriff zwischen unterschiedlichen Medien und verschiedenen Datenquellen ermöglicht.

Zudem bietet Linked Data im raumzeitlichen Kontext die notwendige technische Basis für halbautomatische Validierung und Pflege der Daten. Deep Fact Validation (DeFacto) ${ }^{77}$ kann als Beispiel für eine derartige Anwendungsentwicklung genannt werden, das Statements des Resource Description Framework (RDF) mithilfe von Webinhalten validiert.

Durch den Einsatz semantischer Vorgehensweisen ergeben sich Herausforderungen, an denen sich Bibliotheken beteiligen können um aktiv Forschungsvorhaben zu unterstützen, voranzutreiben und ebenfalls entsprechende Resultate für ihre Services zu nutzen, z.B. eine raumzeitliche Linked-Data-basierte Suchmöglichkeit in einem vernetzten Korpora.

Zusätzlich sind weitere Entwicklungen notwendig, die den Nutzern einen Zugang zu Informationen ohne größere technische Hürden wie der Anwendung einer Abfragesprache wie SPARQL Protocol And RDF Query Language (SPARQL) ermöglichen. Als Beispiel für eine solche Entwicklung kann der Explorer für raumzeitlichen Inhalt $(\mathrm{SPEX})^{78}$ genannt werden, der visuell-gestützte Abfragen für Linked Data im raumzeitlichen Kontext erlaubt. Die Anwendung unterstützt durch vorgeschlagene Klassen, Eigenschaften, Instanzen und dem automatischen Ma-

71 http://libris.kb.se.

72 http://www.dnb.de/DE/Service/DigitaleDienste/LinkedData/link eddata_node.html.

73 http://data.bnf.fr.

$74 \mathrm{http}: / /$ nektar.oszk.hu/wiki/Semantic_web.

75 http://www.bl.uk/bibliografic/datafree.html.

$76 \mathrm{http}: / /$ id.loc.gov.

$77 \mathrm{http}: / /$ defacto.aksw.org.

78 Anwendung unter http://giv-lodum.uni-muenster.de/spex, Code-Repository unter https://github.com/lodum/SPEX. nagement der damit verknüpften raumzeitlichen Informationen, das Generieren von Abfragen. Durch die zusätzliche Wiedergabe der Informationen, per Karten- und Zeitleistenvisualisierung und automatischem Labelling, ermöglicht der Explorer Abfragen ohne die Eingabe einer Linked-Data-spezifischen Syntax durchzuführen. ${ }^{79}$ In diesem Zusammenhang würde auch eine Erschließung der Kartenbestände per Raster-to-Vector-Verfahren in Verbindung mit Feature-Extraktion komplett neue Möglichkeiten eröffnen. ${ }^{80}$

Die Bedeutung und Anwendung von Geodaten im Forschungsbereich wird aktiv in der Forschungs-Community diskutiert und weiterentwickelt, beispielsweise innerhalb der Interessengruppe Geospatial der Research Data Alliance (RDA) ${ }^{81}$ oder der GEO Data Sharing Working Group ${ }^{82}$ innerhalb des Committee on Data for Science and Technology (CODATA). ${ }^{83}$ Daran kann man auch sehr gut die internationale und zugleich die forschungsrelevante Dimension dieses Themenkomplexes erkennen. Auch innerhalb von fachspezifischen Organisationen gibt es Interessengruppen für den Umgang mit raumzeitlichen Daten wie der GeoHumanities Special Interest Group (SIG) ${ }^{84}$ der Alliance of Digital Humanities Organizations (ADHO), ${ }^{85}$ der GeoHumanities Working Group ${ }^{86}$ von DARIAH oder auch die regelmäßigen fachbezogenen Panels auf dem Deutschen Bibliothekartag ${ }^{87}$ oder dem Deutschen Historikertag. ${ }^{88}$

Auch lokale Kooperationsgemeinschaften wie Geo.X ${ }^{89}$ sind ein Beispiel, wie diverse wissenschaftlichen Einrichtungen, u. a. GFZ Potsdam, Humboldt-Universität zu Berlin (HU) und das Museum für Naturkunde (MfN) - LeibnizInstitut für Evolutions- und Biodiversitätsforschung, gemeinsam die Entwicklung von Methoden und Technologien sowie die Bereitstellung von erforderlichen wissenschaftlichen Infrastrukturen mit Bibliotheken, Medienzentren und Serviceleistungen für raumzeitliche Informationen im interdisziplinären Kontext bewerkstelligen können. Auch innerhalb solcher Kooperationsgemeinschaften können Bibliotheken die Digitalisierung und die Entwicklung bezüglich

\footnotetext{
79 Scheider et al. (2014) $146 \mathrm{f}$.

80 Vgl. Chiang (2015).

81 https://www.rd-alliance.org/groups/geospatial-ig.html.

82 http://www.codata.org/geo-data-sharing-working-group.

$83 \mathrm{http}: / /$ www.codata.org.

$84 \mathrm{http}: / /$ geohumanities.org.

$85 \mathrm{http} / / /$ adho.org.

86 https://www.dariah.eu/activities/working-groups/geohumani ties.

87 http://www.bibliothekartag.de.

88 https://www.historikertag.de/Muenster2018.

89 https://www.geo-x.net.
} 


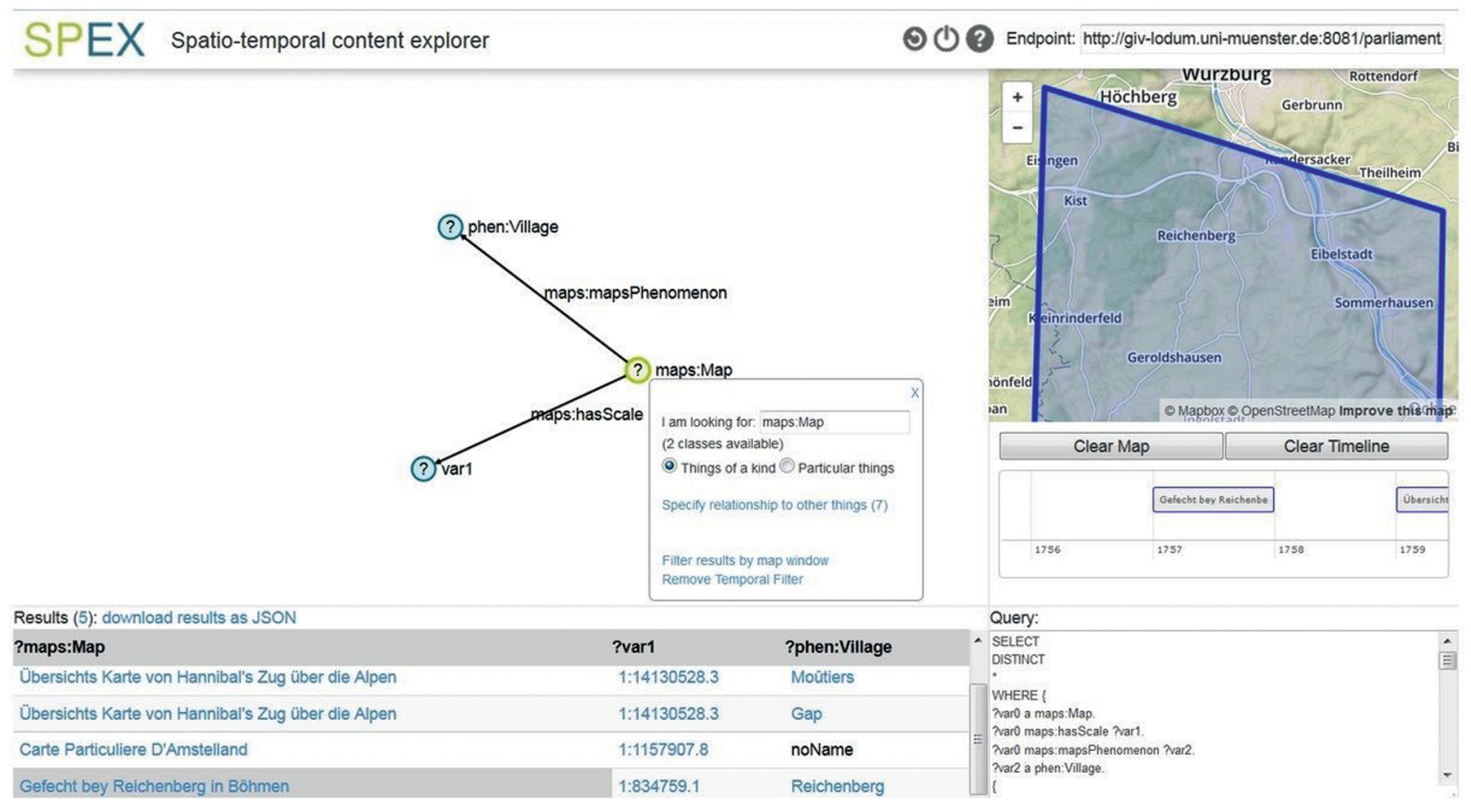

Abb. 2: SPEX ${ }^{90}$

Geoinformationen aus Bibliotheksbeständen aktiv mitgestalten.

\section{Perspektiven}

Auch wenn einzelne Entwicklungen der letzten Jahre unter Verwendung von webbasierten GIS-Entwicklungen, offenen Schnittstellen und Standards einen gezielten Zugriff auf den digitalen Bestand von Geografika in Bibliotheken erlauben, gibt es noch eine Fülle an historisch gewachsenen Anwendungen, die Geografika ausschließlich über textbasierte Suchmethoden mit Attributen wie Titelstichwörter - also auf Ebene der klassischen Metadaten zugänglich machen. Die Einbindung von Funktionen, die GIS-spezifische Datentypen unterstützen und eine kartenbasierte interaktive Suche wie auch Visualisierung ermöglichen, wäre nur ein adäquates Mittel zur Bereitstellung von Geoinformationen, um die Nutzer bei ihren Anliegen zu unterstützen und ihnen Ressourcen effizient zur Verfügung zu stellen.

90 SPEX ermöglicht es dem Benutzer, die Fragestellung im Abfragefenster visuell zu konstruieren. Im Hintergrund werden die vom Benutzer eingegebenen Daten gespeichert und in die SPARQL-Syntax, der Sprache, in der RDF-Datenbanken abgefragt werden, übersetzt. Siehe https://github.com/lodum/SPEX.
Hierbei sollte der Datenzugang für Nutzer möglichst durch benutzerfreundlich gestaltete Suchportale und Anwendungen unterstützt werden. Die Entwicklung von angemessenen Lösungen für forschungsrelevante Anwendungen sind zugleich mit Beratungs-, Weiterbildungs- und Schulungsangeboten zu ergänzen, um die Nutzung von Geoinformationen und die notwendige Vernetzung mit anderen Ressourcen in Bibliotheken zu gewährleisten und $\mathrm{zu}$ verbessern. Nur durch diese Kombination aus verfügbaren und analysierbaren Daten und Sammlungen mit entsprechenden Werkzeugen und Schulungsangeboten können Forschungsvorhaben gezielt ermöglicht und unterstützt werden. Faktisch bedeutet dies, dass entsprechende Personalressourcen durch Bibliothekare, Entwickler, Metadaten-Spezialisten und Geo-Experten zur Verfügung gestellt werden müssen.

\subsection{Datenmanagement}

Um Web-GIS-Anwendungen im bibliothekarischen Kontext $\mathrm{zu}$ betreiben und damit auf eine Vielzahl von Geoinformationen zurückzugreifen, ist es zunächst notwendig, die Sammlungen der Geografika, die bisher mehr oder weniger partiell digitalisiert sind, zu erschließen. Dies schließt auch standortspezifische Bestände wie lokale Buch- und Datenbestände - beispielsweise sind hier historische Adressbücher und Vorlesungsverzeichnisse $\mathrm{zu}$ 
nennen - mit ein. Dies trägt zum Erhalt des Kulturerbes bei und schafft zugleich neue Anreize für die Forschung. Hier ist eine direkte Abstimmung mit den entsprechenden Förderern notwendig bzw. es kann das bereits bestehende Förderungsangebot aktiv zur digitalen Erschließung von archivalischen Quellen genutzt werden.

Neben der Tatsache, dass in Sammlungen nicht alle Geografika digital erschlossen und vollständig georeferenziert vorliegen, sind nicht geografisch referenzierte Karten oftmals in den Anwendungsmöglichkeiten durch unzureichende Verschlagwortung der Ortsangaben beschränkt. ${ }^{91}$ Neben Beispielen von Initiativen der Anreicherung von Metadaten mit Koordinatenangaben wie auch von Normdaten wie durch die Deutsche Nationalbibliothek (DNB), gibt es oftmals in bibliothekarischen Sammlungen noch Werke, die auf eine Weise erschlossen worden sind, die eine Suche und eine anschließende weitere Verwendung nicht intuitiv ermöglichen. Besonders im Bereich der Altkarten findet man hierfür zahlreiche Beispiele, so dass Altkarten oft nur als Kartenwerk verzeichnet und Einzelkarten via Verweise auf die dazugehörigen Indexblätter erschlossen wurden. ${ }^{92}$

Metadaten in denen keine Koordinaten bereitgestellt werden, schränken semantisch nicht eindeutige Ortsnamen und eine nicht detaillierte Kategorisierung die Verwendbarkeit enorm ein. Gerade in diesen Fällen können Werkzeuge zur automatischen Verschlagwortung von Werken und Forschungsdaten mit Geoinformationen auf Basis von Normdateien einen Lösungsansatz bieten. ${ }^{93}$ Die Anreicherung von Ortsnamen kann durch Entwicklungen unterstützt werden, wie dies beim Historischen OrtsNamenVerzeichnis (HONV) ${ }^{94}$ für Mecklenburg-Vorpommern umgesetzt wurde. Dieses stellt ein Ortsnamenverzeichnis mit historischen bzw. lokalen Ortsnamen zur Verfügung und erhöht dadurch die Auffindbarkeit und Vernetzung von Werken in unterschiedlichen Epochen und Kontexten. ${ }^{95}$ Zudem bietet die Anreicherung mit Koordinaten unter Angabe des zugehörigen Referenzsystems einen eindeutigen geografischen Identifier. Generell sollten erfasste geografische Angaben soweit wie möglich in den Metadaten per Geokodierung-Services z.B. mittels

91 Vgl. Mendt (2014).

92 Scheven (2015) 38f.

93 Schöning-Walter (2011) 49f.

94 Historischen OrtsNamenVerzeichnis (HONV) für MecklenburgVorpommern im Rahmen des Projektes Virtuelles Kulturlandschaftslaboratorium (VKLandLab) unter www.vklandlab.uni-rostock.de.

95 Vgl. Walter et al. (2014).

$96 \mathrm{http}: / /$ www.geonames.org.
GeoNames ${ }^{96}$ mit Koordinatenangaben angereichert werden. ${ }^{97}$

Gerade die Bereitstellung von Koordinaten bei Karten wie auch bei Normdaten, ermöglicht effizientere und intuitivere Such- und Auswahlmöglichkeiten per interaktiver Kartenanwendung in Bibliothekskatalogen, wie dies z.B. im Kartenportal der Schottischen Nationalbibliothek umgesetzt wurde. ${ }^{98}$ Eine hilfreiche Suche wertet anhand der dargebotenen Begriffe vorhandene Normdaten aus und berücksichtigt dabei die an den Normdaten erfassten Koordinaten. ${ }^{99}$

Zudem bringen eine detaillierte Auszeichnung und eine Implementierung einer Recherche per geografischer Facette in Katalogen gewisse Vorteile mit sich, wie beispielsweise eine signifikante Erhöhung des Recalls. ${ }^{100}$

Die bisherigen Anwendungen von Kartensammlungen stellen oftmals Kartendigitalisate als Bilddatei zur Verfügung und räumliche Informationen verbleiben auf der Ebene der Metadaten als Koordinaten und Ortsnamen; ein Digitalisierungsgrad, der auch bei gedruckten Werken und Drucken zum jetzigen Zeitpunkt vorzufinden ist. ${ }^{101}$ Der Anwender hat oftmals die Möglichkeit, das Bild zu betrachten bzw. Bild- und Metadaten zu beziehen und diese herunterzuladen. Ein direktes georeferenziertes Arbeiten mit den Daten ist damit allerdings nicht gegeben. Bereits 2001 hatte Peter Keller festgestellt, dass es für eine Kartensammlung essentiell sei, sich neuen digitalen Möglichkeiten zu öffnen, um zukünftig erfolgreich zu sein. ${ }^{102}$ Ein Postulat, dass heute siebzehn Jahre später, oftmals leider eben doch noch als Desiderat bezeichnet werden muss.

Georeferenzierte Karten erlauben in Verbindung mit GIS-Funktionalitäten die Beantwortung wie auch die Generierung von Forschungsfragen. Um von einer bestehenden Kartensammlung, deren Bestand noch nicht georeferenziert vorliegt, zu einem georeferenzierten Bestand zu kommen, gibt es unterschiedliche Ansatzweisen. Um den Ressourceneinsatz für Bibliotheken $\mathrm{zu}$ verringern, gibt es Automatisierungs-Techniken wie die Georeferenzierung mittels Bildbearbeitungsverfahren ${ }^{103}$ und Crowdsourcingbasierte Lösungen. Kartenreferenzierung durch Crowdsourcing-basierte Verfahren stellen eine alternative, effiziente und kostengünstige Lösung dar. Ein positiver Nebeneffekt ist eine aktive Öffentlichkeitsarbeit durch

97 Scheven (2015) $42 \mathrm{ff}$.

98 http://maps.nls.uk/geo/find/\#.

99 Scheven (2016) 33.

100 Vgl. Wiesenmüller (2012).

101 Vgl. Klaffki et al. (2018).

102 Vgl. Keller (2001).

103 Vgl. Röhm et al. (2012). 
Einbeziehung der Nutzer. ${ }^{104}$ Zudem ist die Georeferenzierung durch Fachfremde als verlässlich einzustufen und führt zu einer hohen Genauigkeit der Referenzierung. ${ }^{105} \mathrm{Als}$ erfolgreiche Projekte der letzten Jahre können die Georeferenzierung von historischen Karten der Universitäts- und Landesbibliothek Darmstadt, ${ }^{106}$ der Crowdsouring-Ansatz des Virtuellen Kartenforums $2.0^{107}$ und auch das Georeferenzierungs-Projekt der British Library ${ }^{108}$ genannt werden. Ein weiteres Crowdsourcing-Beispiel ist von der ETH-Bibliothek, die Teile ihres Bildbestandes per Plattform des Smapshot-Projektes ${ }^{109}$ verorten lässt. Die Smapshot-Plattform ermöglicht hierbei die Georeferenzierung von Schweizer Landschaftsbildern im dreidimensionalen Raum.

Für Georeferenzierungen besteht die Möglichkeit für Bibliotheken, auf Open-Source-Software wie QGIS mit dem Gereferenzier-Plugin, ${ }^{110}$ zurückzugreifen bzw. bestehende Services zur Geokodierung bzw. GeoreferenzierungTools $\mathrm{zu}$ verwenden und in die eigenen Anwendungen $\mathrm{zu}$ integrieren. Beispiele für bestehende Entwicklungen zur Georeferenzierung sind der Historic Maps Georeferencer ${ }^{111}$ der ULB Münster, der New York Public Library Map Warper ${ }^{112}$ oder der Harvard University World Map WARP, ${ }^{113}$ die Ihre Entwicklungen in Code-Repositorien wie Github ${ }^{114}$ zur Verfügung stellen und für eine Crowdsourcing-basierte Anwendung adaptiert werden können. Aber auch kommerzielle Angebote wie der Georeferencer ${ }^{115}$ von Klokan Technologies finden in Bibliotheken wie auch auf Portalen wie der David Rumsey Map Collection ${ }^{116}$ Verwendung.

Derzeitige Bibliothekskataloge basieren noch häufig auf Technologien mit wenig Unterstützung für raumzeitlichen Datentypen. ${ }^{117}$ Einen Mehrwert für externe Anwendungen würde durch die Aufbereitung und Bereitstellung von Geodaten mittels offener webbasierter Standards wie Web Map Service (WMS), ${ }^{118}$ Web Feature

104 Ammon (2016) $19 \mathrm{ff}$.

105 Bill und Walter (2015) 179.

$106 \mathrm{https}$ ///www.ulb.tu-darmstadt.de/georeferencer.

107 Vgl. Bill und Walter (2015).

108 https://www.bl.uk/georeferencer/georefabout.html.

109 https://blogs.ethz.ch/crowdsourcing/2017/05/29/smapshot-abwinter-201718-georeferenzieren-im-3d-raum-leicht-gemacht.

$110 \mathrm{https} / /$ docs.qgis.org/2.18/de/docs/user_manual/plugins/plu gins_georeferencer.html.

$111 \mathrm{http} / / /$ linkeddata.uni-muenster.de/georeferencer/georef.html. $112 \mathrm{http} / / /$ maps.nypl.org/warper.

$113 \mathrm{http}: / /$ warp.worldmap.harvard.edu.

$114 \mathrm{https}$ //github.com.

$115 \mathrm{https} / / / \mathrm{www}$.georeferencer.com/institutions.

116 https://www.davidrumsey.com/view/georeferencer.

117 Scheider et al. (2014) 140.

$118 \mathrm{http} / / /$ www.opengeospatial.org/standards/wms.
Service (WFS) ${ }^{119}$ und Web Coverage Service (WCS) ${ }^{120}$ generiert werden, die es erlauben, georeferenzierte Bildbzw. Vektordaten direkt in die Anwendung durch entsprechende Schnittstelle einzubinden und wiederzuverwenden. Als Beispiel kann das Virtuelle Kartenforum 2.0 genannt werden, das Karten des Deutschen Reiches als WMS $^{121}$ und WCS $^{122}$ unter freier Lizenz zur Verfügung stellt. ${ }^{123}$

Derzeitige Bibliothekskataloge basieren oftmals auf Technologien ohne semantische Verbindungen bzgl. raumzeitlichen Informationen, Inhalt und Kontext. Falls die Identifier zudem unpubliziert bleiben, entstehen mit den Katalogen Datensilos ohne semantische Vernetzung der Inhalt und geteiltem Hintergrundwissen. ${ }^{124}$

Aktuelle Initiativen wie der Aufbau einer Linked Open Data-Plattform an der Österreichischen Nationalbibliothek können in diesem Zusammenhang genannt werden. Hier werden Daten der Kataloge mit Daten zu Personen und geografischen Orten verknüpft zur Verfügung gestellt. ${ }^{125}$

\subsection{Applikationen}

Mit der Bereitstellung von Web-GIS-Lösungen, die es erlauben eigene raumzeitliche Forschungsdaten zu visualisieren und zu analysieren, können Bibliotheken gezielt den Anforderungen von Forschung und Wissenschaft gerecht werden.

Bisherige Anwendungen sind aber oftmals als eigenständige Lösungen konzipiert worden, somit können GeoRessourcen von Bibliotheken wie spezielle historische Karten-Kollektionen begrenzt und durch Mehraufwand mit den eigenen Forschungsdaten in der Anwendung verschnitten werden. Als Beispiele können hier der DARIAHDE-Geo-Browser und Palladio von Stanford und Humanities + Design ${ }^{126}$ genannt werden. Wünschenswert wären hier zentrale Lösungen, die auf der einen Seite als Kartenportal zum Recherchieren von Bibliotheksbeständen dienen und auf der anderen Seite Nutzer die Verwaltung von eigenen raumzeitlichen Forschungsdaten erlauben.

\footnotetext{
119 http://www.opengeospatial.org/standards/wfs. $120 \mathrm{http}: / /$ www.opengeospatial.org/standards/wcs. $121 \mathrm{http}: / /$ kartenforum.slub-dresden.de/cgi-bin/tkows?SERVICE=W MS\&REQUEST $=$ GetCapabilities\&VERSION=1.0.0.

$122 \mathrm{http} / / /$ kartenforum.slub-dresden.de/cgi-bin/tkows?SERVICE=W CS\&REQUEST $=$ GetCapabilities\&VERSION=1.0.0.

$123 \mathrm{https} / / /$ kartenforum.slub-dresden.de/vkviewer/static/service.

124 Scheider et al. (2014) 140.

125 Schmidt (2018) 140.

$126 \mathrm{http}: / /$ hdlab.stanford.edu/palladio.
} 
Neben den ersten Ansätzen, Geodaten-Portale an Campussen für die verschiedenen Fachbereiche zu schaffen, können verstärkt zentrale Dienste generiert werden, die georelevante Bestandsdaten wie Kartensammlungen mit weiteren universitären wissenschaftlichen Sammlungen, die digitalisiert und mit notwendigen Geoinformationen erschlossen sind, koppelt und diese dem Nutzer verknüpft zur Recherche und Analyse zur Verfügung stellt. Denkbar wäre, dass hierbei neben georeferenzierten Karten als Overlay ebenfalls weitere Informationen aus Literatur- und Medieninhalten zum Einsatz kommen. Ein mögliches Szenario wäre die Selektion einer spezifischen Region einer historischen Karte, um diesbezüglich weitere Abfragen durchzuführen, die Zugang zu allen weiteren verfügbaren Medien für diese Region und zu der relevanten Zeit erlauben.

Ein Projektbeispiel für eine vernetzte Anwendung von heterogenen Sammlungsdaten ist das Virtuelle Kartenlabor (GlobMapLab) als Zugang zur Sammlung Perthes der Universität Erfurt, das Karten, Archivmaterial und Informationen über beteiligten Personen verknüpft recherchierbar macht, präsentiert und eine Analyse der Karten mit Kontextobjekten wie Literatur und Archivalien ermöglicht. ${ }^{127}$

\subsection{Support und Beratung}

Um dem wachsenden Bedarf an fachkompetenter Beratung im Bereich Geoinformation und dessen Anwendungen entgegenzukommen, müssen Bibliotheken aufgrund Ihrer zentralen Rolle eigene Beratungsservices offerieren und als Vermittler zu externen Geodaten-Dienstleistern fungieren. ${ }^{128}$ Ergänzend zu dem Angebot von Anwendungen können Bibliotheken Entwicklungs-Support für räumliche Anwendungen als neue Service-Komponente in ihr Portfolio aufnehmen. ${ }^{129}$ Effektives Marketing, einschließlich Sichtbarkeit und Langlebigkeit von Webseiten, ist ein effektives Werkzeug, um das Bewusstsein für GIS-Dienstleistungen zu erhöhen. Um einen Support für Geoinformationen zu etablieren, ist es allerdings notwendig, die erforderlichen Rahmenbedingungen zu schaffen.

Hierbei müssen Bibliotheksleitungen im Zusammenspiel mit den Abteilungen am Campus Lösungen für die technische, personelle und finanzielle Ressourcen-Frage

127 Vgl. Henniges et al. (2017).

128 Vgl. Researcher Support der North Carolina State University Library (Data\&GIS) https://www.lib.ncsu.edu/services/data-visualiza tion.

129 Godfrey (2016) 31. erarbeiten, um den Betrieb langfristig realisieren zu können. ${ }^{130}$ Neue Dienste und bibliothekarische Angebote benötigen dauerhafte Ressourcen, um gegenüber der wissenschaftlichen Community bzw. dem eigenen Campus ein aus technischen Komponenten, aus fachlicher Expertise und den entsprechenden Beratungsangeboten kombiniertes Serviceangebot dauerhaft anbieten zu können. ${ }^{131}$ Fachleute mit fundierten GIS-relevanten Kenntnissen sind hierbei unabdingbar, um die Verknüpfung zwischen Geoinformations-Technologien und Bibliotheks-Services umzusetzen und zu forcieren.

Als Beispiel für einen umfassenden Support kann das Beratungsangebot der Watson Library, Kansas University $^{132}$ genannt werden, der nicht nur Hilfestellung zu Sammlungs-spezifischen Anliegen bietet, sondern auch zu allgemeinen Fragen bzgl. Geodaten, Geoanwendungen wie auch räumlichen Analysemöglichkeiten.

\section{Schlussbemerkung}

Die gesteigerte Nutzung von GIS-Anwendungen, besonders im Bereich von Forschung und Lehre, kreiert einen erhöhten Bedarf an Geodaten und den dafür erforderlichen Softwareanwendungen mit Support- und Beratungsangeboten. Wissenschaftliche Bibliotheken haben hierfür die relevanten Bestände und Sammlungen und sind aufgrund ihrer zentralen Stellung als Informationseinrichtungen und wissenschaftlicher Dienstleister vor Ort bestens dafür geeignet, den Bedarf an Geoinformationen und den damit verbundenen Services für ihren Campus und ihre wissenschaftlichen Communities abzudecken. Einige dieser bereits existierenden Dienste und Angebote haben wir aufgezeigt und zugleich verdeutlicht, dass hier vielfältige Nachnutzungsmöglichkeiten existieren.

In bisherigen Veröffentlichungen zu Geodaten an Bibliotheken ist oftmals geäußert worden, dass es die primäre Aufgabe von Bibliotheken sei, diese in einer Form und Formaten bereitzustellen, damit sie in GIS-Anwendungen genutzt werden können. Die von uns beschriebenen Aspekte von etablierten Diensten und Services zeigen aber auch in aller Deutlichkeit, dass oftmals zeitlich begrenzte Projekte mit konkretem Forschungsinteresse der Rahmen sind, in dem diese Anwendungen entwickelt werden. $\mathrm{Zu}$ -

130 Wonke-Stehle und Christof (2014) $18 \mathrm{f}$.

131 Ein Beispiel für solch ein gelungenes und etabliertes Konzept ist die Göttinger eResearch Alliance, die gemeinsam von der SUB Göttingen und dem Rechenzentrum (GWDG) betrieben wird, siehe http:// www.eresearch.uni-goettingen.de.

132 https://lib.ku.edu/gis, https://lib.ku.edu/gis/lab. 
gleich können diese GIS-Technologien und -Softwarelösungen schon bei der Bereitstellung von Geoinformationen im Kontext von generischen Anforderungen in und von Bibliotheken gewinnbringend eingesetzt werden, etwa in Form von Recherche-Portalen mit Visualisierungsund Analysefunktionen. Das eine schließt das andere nicht aus, aber es zeigt auch, dass eine Vielzahl an Möglichkeiten existieren, die über das bloße Zurverfügungstellen von Geodaten hinausgehen und oftmals konkrete Forschungsinteressen dazu führen, dass generisch nachnutzbare Werkzeuge und Datensammlungen entstehen, die das Serviceangebot von Bibliotheken nachhaltig erweitern können.

Die Bereitstellung der Daten, mittels State-of-the-ArtTechnologien, wie dem Einsatz von offenen Webstandards, beispielsweise durch OGC-Web-Services, ist zugleich unerlässlich, um Nutzern mit möglichst geringen Barrieren einen Zugang zu dem Informations-Schatz der Bibliotheken zu bieten und eine effektive Nutzung der Daten zu gewährleisten. Es ist allerdings auch festzustellen, dass kein universell gültiger Ansatz für die Realisierung von GIS-Services an Bibliotheken existiert. Das Design und die Umsetzung der Services ist individuell durch die Sammlungsbestände und die lokalen Bedingungen geprägt, abhängig von der verfolgten Strategie der Bibliothek und deren Universität, fundiert auf den Möglichkeiten der vorhandenen technischen Infrastruktur, der finanziellen und personellen Ressourcen und den unterschiedlichen Anforderungen des jeweiligen Campus und der fachwissenschaftlichen Communities.

Geoinformation-Services in Bibliotheken haben sich somit in den vergangenen Jahren in unterschiedlicher Geschwindigkeit und Ausprägung entwickelt: So werden sowohl Geoinformationen mittels Web-GIS bereitgestellt und dadurch eine Nachnutzung und Integration in externe Anwendungen aufgrund genutzter Standards gewährleistet als auch Geografika nur analog verwaltet. Es ist zu registrieren, dass eine Ungleichheit des Gleichzeitigen vorherrscht - ein Phänomen, das sicherlich auch in den kommenden Jahren noch vorzufinden sein wird, aber es wäre wünschenswert, wenn die bereits von einigen Bibliotheken eingeschlagenen Wege, von anderen erprobt und adaptiert werden würden.

Festzuhalten bleibt, dass es unabdingbar ist, dass Bibliotheken mit den Anforderungen durch Anwendung von Data Science Technologien wie Data Mining und Machine Learning wachsen und ihre Informationen im Einklang mit den Ansprüchen ihrer bisherigen und zukünftigen Nutzer bereitstellen. Einerseits kann dies durch die (infra)-strukturelle Integration von existierenden GIS-Services gelingen, andererseits muss dies in enger Abstimmung und
Kooperation mit den Fachwissenschaftlern vor Ort bzw. fachwissenschaftlichen Communities geschehen, so dass konkrete Dienste etabliert und dauerhaft anforderungsbasiert weiterentwickelt werden. Denn digitale Systeme wie auch digital erschlossene Sammlungen benötigen eine strukturierte und dauerhafte Weiterentwicklung und Pflege. Zugleich muss gewährleistet sein, dass die mit diesen Systemen durchgeführten Analysen und Visualisierungen und die zugrundeliegenden Daten, durch offene Lizenzen frei genutzt werden können. Nur dann ist sichergestellt, dass solch ein Portfolio auch zu der digitalen Transformation einer Bibliothek beitragen kann.

\section{Literaturverzeichnis}

Alex, Beatrice; Byrne, Kate; Grover, Claire; Tobin, Richard (2015): Adapting the Edinburgh geoparser for historical georeferencing. In: International Journal of Humanities and Arts Computing, 9 (1), 15-35. Verfügbar unter doi:10.3366/ijhac.2015.0136.

Ammon, Michael (2016): „Ich wünsche mir sehr gerne noch weitere Karten ..." Georeferenzierung von historischen Karten mittels Crowdsourcing an der Universitäts-und Landesbibliothek Darmstadt. In: AKMB-news: Informationen zu Kunst, Museum und Bibliothek, 22 (2), 17-24.

Bill, Ralf; Walter, Kai (2015): Crowdsourcing zur Georeferenzierung alter topografischer Karten-Ansatz, Erfahrungen und Qualitätsanalyse. In: Zeitschrift für Geodäsie, Geoinformation und Landmanagement, (eingereicht, DOI 10.12902/zfv-0060-2015).

Ceynowa, Klaus (2018): Research Library Reloaded? Überlegungen zur Zukunft der geisteswissenschaftlichen Forschungsbibliothek. In: Zeitschrift für Bibliothekswesen und Bibliografie 65 (1), 3-7.

Chiang, Yao-Yi (Hrsg.) (2015): Querying historical maps as a unified, structured, and linked spatiotemporal source: vision paper. In: Proceedings of the 23rd SIGSPATIAL International Conference on Advances in Geographic Information Systems. ACM. 16.

Dear, Michael; Ketchum, Jim; Luria, Sarah; Richardson, Douglas (2011): Introducing the geohumanities. In: GeoHumanities, 21-22.

Godfrey, Bruce (2016): Geospatial Data on Your Own Terms. In: PNLA Quarterly, 80 (2), 29-38.

Goodchild, Michael F. (2004): The Alexandria Digital Library Project: review, assessments and prospects. In: D-Lib Magazine, 10 (5). Verfügbar unter www.dlib.org/dlib/may04/goodchild/05good child.html.

Goodchild, Michael F.; Hill, Linda L. (2008): Introduction to digital gazetteer research. In: International Journal of Geographical Information Science, 22 (10), 1039-44.

Gradmann, Stefan (2014): From containers to content to context: The changing role of libraries in eScience and eScholarship. In: Journal of documentation, 70 (2), 241-60.

Henniges, Norman; Rau, Susanne; Smolarski, René; Tzschach, Heiko (2017): Mehr als nur Karten. Das Virtuelle Kartenlabor (GlobMapLab) als Zugang zur Sammlung Perthes. In: Zeitschrift für digitale Geisteswissenschaften. Verfügbar unter DOI: 10.17175/ 2016_001. 
Horstmann, Wolfram; Jahn, Najko; Schmidt, Birgit (2015): Der Wandel der Informationspraxis in Forschung und Bibliothek. In: Zeitschrift für Bibliothekswesen und Bibliografie, 62 (2), 73-79.

Janée, Greg (Hrsg.) (2009): Preserving Geospatial Data: The National Geospatial Digital Archive‘s Approach. In: Archiving Conference, 1, 25-29. Society for Imaging Science and Technology.

Keller, C. Peter (2001): The Map library's Future revisited: A response. In: Cartographic Perspectives, 40, 9-11.

Klaffki, Lisa; Schmunk, Stefan; Stäcker, Thomas (2018): Stand der Kulturgutdigitalisierung in Deutschland. Eine Analyse und Handlungsvorschläge des DARIAH-DE Stakeholdergremiums, Wissenschaftliche Sammlungen'. In: DARIAH-DE Working Papers Nr. 26. Göttingen: DARIAH-DE. Verfügbar unter URN: urn:nbn:de:gbv:7dariah-2018-1-3.

Leidner, Jochen L.; Sinclair, Gail; Webber, Bonnie (Hrsg.) (2003): Grounding spatial named entities for information extraction and question answering. Association for Computational Linguistics.

Martins, Bruno; Anastácio, Ivo; Calado, Pável (2010): A machine learning approach for resolving place references in text. In: Geospatial thinking, 221-36.

Mendt, Jacob (2014): Using FOSS4G in historic map collections. Verfügbar unter http://europe.foss4g.org/2014/sites/default/ files/05-Mendt_0.pdf.

Michalec, Mychaelyn; Welsh, Teresa S. (2007): Quantity and authorship of GIS articles in library and information science literature, 1990-2005. In: Science \& technology libraries, 27 (3), 65-77.

Muratori, Ilaria; Samakovlija, Marcella (2015): Sharing geo data through university libraries: the case of Politecnico di Milano. Verfügbar unter http://geomatica.como.polimi.it/workbooks/n1 2/FOSS4G-eu15_submission_43.pdf.

Overell, Simon; Rüger, Stefan (2008): Using co-occurrence models for place name disambiguation. In: International Journal of Geographical Information Science, 22 (3), 265-87.

Pinol, Jean-Luc (2018): New assets for urban history. In: The Routledge Companion to Spatial History. London: Routledge.

Röhm, Patric; Herold, Hendrik; Meinel, Gotthard (2012): Automatische Georeferenzierung gescannter deutscher Topografischer Karten im Maßstab 1:25.000. In: Kartografische Nachrichten, 62, 195-99.

Scheider, Simon; Degbelo, Auriol; Kuhn, Werner; Przibytzin, Holger (2014): Inhalts-und Kontextbeschreibung. Neuartige Informationsdienste für Bibliotheken mit Linked Spatio-Temporal Data. Verfügbar unter https://gispoint.de/artikelarchiv/gis/2014/ gisscience-ausgabe-42014/3439-inhalts-und-kontextbeschreibung-neuartige-informationsdienste-fuer-bibliotheken-mitlinked-spatio-temporal-data.html.

Scheven, Esther (2015): Geokoordinaten in Bibliotheksdaten. Grundlage für innovative Nachnutzung. In: o-bib. Das offene Bibliotheksjournal, 2 (1), 35-46.

Scheven, Esther (2016): Geografische Koordinaten und Bibliotheksdaten - Regelwerke und Anwendungen. In: Dialog mit Bibliotheken, 28 (1), 30-38.

Schmidt, Alfred (2018): Neue Online-Aktivitäten der Österreichischen Nationalbibliothek. In: BIBLIOTHEK - Forschung und Praxis, 42 (1), 136-40.

Simon, Rainer; Barker, Elton; Isaksen, Leif; Soto Cañamares, Pau de (2015): Linking early geospatial documents, one place at a time: annotation of geographic documents with Recogito. In: e-Perimetron, 10 (2), 49-59.
Schöning-Walter, Christa (2011): Automatische Erschließung Herausforderung und Chance. In: Dialog mit Bibliotheken, 23 (2), 49-51.

Walter, Kai; Labahn, Karsten; Bill, Ralf; Zepf, Robert (2014): Kulturhistorische Forschung und Geodatenportale - raumbasierte Recherche in kulturhistorischen Datenbeständen mithilfe einer generisch erweiterbaren Diensteinfrastruktur. In: Angewandte Geoinformatik, hg. v. Joseph Strobl, Thomas Blaschke, Gerald Griesebner und Bernhard Zagel, 719-28.

Weimer, Katherine H.; Olivares, Miriam; Bedenbaugh, Robin A. (2012): GIS day and web promotion: Retrospective analysis of US ARL libraries' involvement. In: Journal of Map \& Geography Libraries, 8 (1), 39-57.

Wiesenmüller, Heidrun (2012): Die geografische Facette: eine neuartige Recherche-Option für Suchmaschinenkataloge. Konferenzveröffentlichung (Vortragsfolien) auf dem 101. Deutschen Bibliothekartag in Hamburg. Verfügbar unter https:// opus4.kobv.de/opus4-bib-info/frontdoor/index/index/year/ 2012/docld/1139.

Won, Miguel; Murrieta-Flores, Patricia; Martins, Bruno (2018): ensemble named entity recognition (ner): evaluating ner Tools in the identification of Place names in historical corpora. In: Frontiers in Digital Humanities, 5 (2), 1-12.

Wonke-Stehle, Jens; Christof, Jürgen (2014): Fachportale von Bibliotheken. In: BIBLIOTHEK - Forschung und Praxis, 38 (1), 14-19.

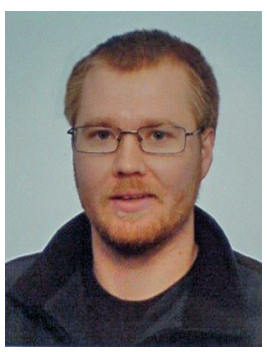

\section{Friedrich Müller}

Niedersächsische Staats- und Universitätsbibliothek Göttingen Abteilung Forschung und Entwicklung Platz der Göttinger Sieben 1 D-37073 Göttingen friedrich.mueller@sub.uni-goettingen.de https://orcid.org/0000-0002-0762-7565

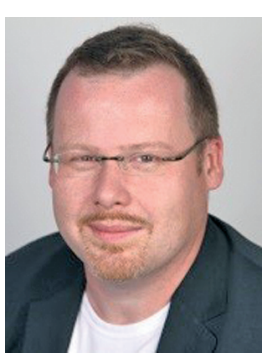

\section{Stefan Schmunk}

University of Applied Sciences Darmstadt Department of Media

Max-Planck-Str. 2

D-64807 Dieburg stefan.schmunk@h-da.de https://orcid.org/0000-0001-9706-9757 